OPEN ACCESS

Edited by:

Martin Burtscher,

University of Innsbruck, Austria

Reviewed by:

Beat Knechtle,

University Hospital Zurich, Switzerland

Susan V. Brooks,

University of Michigan, United States

${ }^{*}$ Correspondence:

Alessandro Moura Zagatto

azagatto@yahoo.com.br

Specialty section:

This article was submitted to

Exercise Physiology,

a section of the journal

Frontiers in Physiology

Received: 01 September 2018

Accepted: 14 March 2019

Published: 10 April 2019

Citation:

de Poli RAB, Roncada LH,

Malta EdS, Artioli GG, Bertuzzi $R$ and

Zagatto AM (2019) Creatine

Supplementation Improves

Phosphagen Energy Pathway During

Supramaximal Effort, but Does Not

Improve Anaerobic Capacity or

Performance. Front. Physiol. 10:352.

doi: 10.3389/fphys.2019.00352

\section{Creatine Supplementation Improves Phosphagen Energy Pathway During Supramaximal Effort, but Does Not Improve Anaerobic Capacity or Performance}

\author{
Rodrigo de Araujo Bonetti de Poli ${ }^{1,2}$, Luan Henrique Roncada ${ }^{1,3}$, Elvis de Souza Malta ${ }^{1,2}$, \\ Guilherme Giannini Artiolit,5, Rômulo Bertuzzi ${ }^{5}$ and Alessandro Moura Zagatto ${ }^{1,2,3 *}$ \\ 1 Laboratory of Physiology and Sport Performance (LAFIDE), São Paulo State University (UNESP), Bauru, Brazil, \\ ${ }^{2}$ Post-Graduate Program in Movement Sciences, São Paulo State University (UNESP), Bauru, Brazil, ${ }^{3}$ Department \\ of Physical Education, School of Science, São Paulo State University (UNESP), Bauru, Brazil, ${ }^{4}$ Applied Physiology \\ and Nutrition Research Group, University of São Paulo (USP), São Paulo, Brazil, ${ }^{5}$ School of Physical Education and Sport, \\ University of São Paulo (USP), São Paulo, Brazil
}

This study aimed to investigate the effects of short-duration creatine monohydrate supplementation on anaerobic capacity (AC), anaerobic energy pathways, and timeto-exhaustion during high-intensity running. Fourteen healthy men underwent a graded exercise test (GXT) followed by a $\dot{\mathrm{V}} \mathrm{O}_{2 \max }$ confirmation test, 5 submaximal efforts, and 4 supramaximal running bouts at $115 \%$ of $\dot{\mathrm{V}} \mathrm{O}_{2 \max }$ intensity (the first two supramaximal sessions were applied as familiarization trials) to measure the AC using two procedures; the maximum accumulated oxygen deficit (MAOD) and non-oxidative pathways energetics sum ( $\left.\mathrm{AC}_{[\mathrm{La}-]+E P O C f a s t}\right)$. The investigation was conducted in a single-blind and placebo-controlled manner, with participants performing the efforts first after being supplemented with a placebo (dextrose $20 \mathrm{~g} \cdot$ day $^{-1}$ for 5 days), and then, after a 7 day "placebo" washout period, they started the same procedure under creatine supplementation (20 g.day ${ }^{-1}$ for 5 days. This order was chosen due to the prolonged washout of creatine. MAOD was not different between placebo (3.35 $\pm 0.65 L)$ and creatine conditions ( $3.39 \pm 0.79 L ; P=0.58)$ and presented $a$

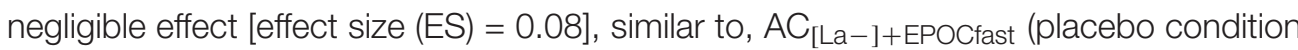
(3.66 \pm 0.79 Land under creatine ingestion $3.82 \pm 0.85 \mathrm{~L} ; \mathrm{P}=0.07$ ) presenting a small effect $(E S=0.20)$. The energetics from the phosphagen pathway increased significantly after creatine supplementation $(1.66 \pm 0.40 \mathrm{~L})$ compared to the placebo condition (1.55 $\pm 0.42 \mathrm{~L} ; \mathrm{P}=0.03)$. However, the glycolytic and oxidative pathways were not different between conditions. Furthermore, time to exhaustion did not differ between placebo (160.79 $\pm 37.76 \mathrm{~s}$ ) and creatine conditions (163.64 $\pm 38.72 ; \mathrm{P}=0.49$ ). Therefore, we can conclude that creatine supplementation improves the phosphagen energy contribution, but with no statistical effect on AC or time to exhaustion in supramaximal running.

Keywords: creatine, anaerobic capacity, energy contribution, high-intensity effort, performance 


\section{INTRODUCTION}

Creatine ( $\alpha$-methyl guanidine-acetic acid) is a nitrogen amine which can be obtained in diet (e.g., red and fish meat) and endogenously synthesized by the liver, kidneys, and pancreas (Hall and Trojian, 2013) and it is predominantly stored in skeletal muscle $(\approx 95 \%)$ in both its free and phosphorylated forms (i.e., phosphorylcreatine) (Persky and Brazeau, 2001). One of the major roles of creatine is to act as a non-mitochondrial energy buffer, rapidly transferring energy through a reversible reaction catalyzed by the creatine kinase enzyme (Gualano et al., 2010).

Short-term creatine monohydrate supplementation has been widely used to improve performance in high-intensity and short-term efforts in cycling (Jacobs et al., 1997; Volek and Rawson, 2004; Hall and Trojian, 2013). Its effects have been mainly associated with increased intramuscular stores of creatine $(\sim+20 \%)$ (Harris et al., 1992) and increased phosphorylcreatine resynthesis rate (Greenhaff et al., 1994). Since creatine supplementation can significantly increase phosphorylcreatine intramuscular stores, it has been shown to improve the energy supply from the phosphagen systems (ePCr) (Yquel et al., 2002; Bemben and Lamont, 2005), thereby increasing the maximum capacity to resynthesize adenosine triphosphate (ATP) by non-oxidative pathways [i.e., anaerobic capacity $(\mathrm{AC})$ ] during high-intensity exercise. These changes could ultimately lead to improved performance in this type of exercise (Doherty et al., 2000).

Jacobs et al. (1997) verified the effects of short-duration creatine supplementation $\left(20 \mathrm{~g} \cdot \mathrm{day}^{-1}\right.$ for 5 days $)$ on AC, measured using the maximal accumulated oxygen deficit (MAOD), and in supramaximal effort performance at $125 \%$ of the maximal oxygen uptake $\left(\mathrm{V}_{2 \max }\right)$. These authors reported an increase of $\approx 9 \%$ in MAOD and $\approx 8 \%$ in timeto-exhaustion following supplementation. However, although it is a well-accepted measure of AC, MAOD does not allow for the isolated estimation of ePCr and has poor reliability (i.e., high limits of agreement). This may have hindered the detection of small differences in the anaerobic metabolism (Doherty et al., 2000, 2002).

Some studies (Bertuzzi et al., 2010; Zagatto et al., 2016; Miyagi et al., 2017) have proposed an alternative method to estimate the $\mathrm{AC}$, denominated alternative MAOD. This method determines the AC through the sum of energetic equivalents of the net blood lactate accumulated during exercise and the fast component of excess post-exercise oxygen consumption $\left(\mathrm{EPOC}_{\text {fast }}\right.$ ), which allows estimation of the contribution from the glycolytic $\left(\mathrm{e}\left[\mathrm{La}^{-}\right]\right)$and ePCr pathways, respectively. However, the alternative MAOD does not measure oxygen deficit, thus we will call it AC estimated by the nonoxidative energetics sum measured during a single supramaximal effort $\left(\mathrm{AC}_{[\mathrm{La}-]+\mathrm{EPOCfast}}\right)$. Some studies have demonstrated that $\mathrm{AC}_{[\mathrm{La}-]+\mathrm{EPOCfast}}$ is not different and is significantly correlated with conventional MAOD determined during running and cycling (Bertuzzi et al., 2010; Zagatto et al., 2016, 2017b; Miyagi et al., 2017), furthermore, $\mathrm{AC}_{[\mathrm{La}-]+\mathrm{EPOCfast}}$ is reliable and sensitive to discriminate individuals with distinct training levels (Zagatto et al., 2017b). Previous studies have also shown that $\mathrm{AC}_{[\mathrm{La}-]+\mathrm{EPOCfast}}$ can be sensitive to detect small changes in the anaerobic metabolism with intake of ergogenic supplements, such as those brought about by caffeine and sodium bicarbonate ingestion (Brisola et al., 2015; de Poli et al., 2016). Together, these findings suggest that $\mathrm{AC}_{[\mathrm{La}-]+\mathrm{EPOCfast}}$ could be more sensitive to detect small changes when compared to conventional MAOD.

Therefore, the study aimed investigate the effects of shortduration creatine monohydrate supplementation on $\mathrm{AC}$ measured by $\mathrm{AC}_{[\mathrm{La}-]+\mathrm{EPOCfast}}$ and $\mathrm{MAOD}$, and on anaerobic energy pathways (i.e., ePCr and e $\left[\mathrm{La}^{-}\right]$), and time-to-exhaustion during high-intensity running. Since it is well documented that creatine supplementation can increase intramuscular phosphorylcreatine, we hypothesized it could increase the contribution of the phosphagen metabolism (i.e., ePCr) during high-intensity exercise, therefore improving performance and AC. This study makes progress in the current literature by investigating the effect of creatine supplementation through a novel method to estimate the AC and, particularly, the effects on non-mitochondrial pathway estimation, which has been hypothesized but not scientifically reported until the current date.

\section{MATERIALS AND METHODS}

\section{Participants}

Eighteen male volunteers were initially enrolled in the study; however, four were excluded, thus, fourteen men [mean \pm SD; age $24 \pm 4$ years; height $173.8 \pm 6.2 \mathrm{~cm}$; total body mass $73.4 \pm 7.4 \mathrm{~kg}]$ completed participation in the study. To be included, volunteers were required to be recreationally active, participate in exercise activities such as running, cycling, and soccer at least 2 times per week, and not have used ergogenic supplements such as beta-alanine and creatine, among others, for at least 6 months. Volunteers who were regularly absent from the trials or presented injuries were excluded from the study.

The volunteers were instructed not to ingest alcohol, caffeine, and sodium bicarbonate and not to perform strenuous exercise $24 \mathrm{~h}$ before each trial. Volunteers were also verbally informed about the experimental procedures and signed an informed consent prior to beginning the study. All experimental procedures were approved by the Human Research Ethics Committee from the School of Sciences, São Paulo State University - UNESP (protocol number: 61323916.5.0000.5398) and the study was conducted in accordance with the Declaration of Helsinki.

\section{Experimental Design}

The investigation was conducted in a single-blind, placebocontrolled, crossover trial. Tests were performed on a motorized treadmill (ATL, Inbramed, Porto Alegre, Brazil) with a fixed gradient of $1 \%$ (Jones and Doust, 1996), using a safety belt to avoid accidental falls and to allow for maximal effort. In addition, the treadmill had been previously calibrated according to Padulo et al. (2014). Firstly, the volunteers performed a graded exercise test (GXT) to assess $\dot{\mathrm{V}} \mathrm{O}_{2 \max }$ and the lowest running speed 
associated with $\dot{\mathrm{V}} \mathrm{O}_{2 \max }\left(\mathrm{i} \dot{\mathrm{V}} \mathrm{O}_{2 \max }\right)$, followed by four sessions on different days of supramaximal constant workload tests at $115 \%$ of $\mathrm{iVO}_{2 \max }$ to assess $\mathrm{AC}_{[\mathrm{La}-]+\text { EPOCfast }}$ and oxygen uptake $\left(\dot{\mathrm{VO}}_{2}\right)$ integral area (Zagatto et al., 2016; Miyagi et al., 2017). The first two supramaximal sessions served as familiarization trials. The third supramaximal test was performed under the placebo condition and the fourth test under the creatine condition (12 days after the placebo test). We opted to use the singleblind design with treatment order not being counterbalanced due to the long wash-out period necessary for muscle creatine to return to pre-supplementation values (Hultman et al., 1996). The experimental design of the study is presented in Figure 1.

In addition, 5 submaximal efforts were performed as warmups and used to construct the linear regression to allow determination of MAOD. All exhaustive sessions were separated by a minimum interval of $48 \mathrm{~h}$. All participants were verbally encouraged to perform their maximal efforts in all sessions, and all tests were performed at the same time of day to avoid circadian variations in performance and AC (Hill, 2014).

\section{Physiological and Metabolic Measurements}

Respiratory variables were measured breath-by-breath using a gas analyzer (Quark PFT, COSMED, Rome, Italy) calibrated following the manufacturer's instructions. Heart rate was monitored using a transmission belt connected to the gas analyzer (Wireless HR 138 Monitor, COSMED, Rome, Italy). The respiratory variables and heart rate variables were smoothed every 5 points and interpolated second by second to reduce "noise" and increase the reliability of the parameter estimation, as previously reported for assessing MAOD and $\mathrm{AC}_{[\mathrm{La}-]+\text { EPOCfast }}$ (Zagatto et al., 2016; Miyagi et al., 2017).

Blood samples were collected from the earlobe $(25 \mu \mathrm{L})$ at rest (i.e., before the supramaximal effort) to measure the baseline blood lactate concentration ([La- $\left.]_{B L}\right), 3$ and 5 min after the GXT, and 3, 5, and $7 \mathrm{~min}$ after the supramaximal tests to determine the peak blood lactate concentration $\left([\mathrm{La}-]_{\text {peak }}\right)$. Blood samples were stored at $-20^{\circ} \mathrm{C}$ in tubes containing $50 \mu \mathrm{L}$ of $1 \%$ sodium fluoride, being subsequently analyzed in duplicate [Standard Error $0.27 \mathrm{mmol} \cdot \mathrm{L}^{-1} ; \Delta \%-3.84$ (CI95\% -2.96 to -5.33$)$ ] using an automated analyzer (Yellow Springs Instruments model 2900, $\mathrm{OH}$, United States).

\section{Graded Exercise Test (GXT) and Maximal Oxygen Uptake $\left(\mathrm{V}_{2 \max }\right)$ Determination}

The GXT was designed to last $\sim 8-12 \mathrm{~min}$, according to guidelines to assess the $\dot{\mathrm{V}}{ }_{2 \max }$ and $\mathrm{iVO}_{2 \max }$ (Howley et al., 1995). The GXT started at $8 \mathrm{~km} \cdot \mathrm{h}^{-1}$, with $1.5 \mathrm{~km} \cdot \mathrm{h}^{-1}$ increments every 2 min until exhaustion (Brisola et al., 2015; de Poli et al., 2016; Zagatto et al., 2016). Exhaustion was defined as the incapacity to maintain exercise intensity. After GXT, the participants remained for $5 \mathrm{~min}$ in passive recovery, and then returned to the treadmill to run until exhaustion at a workload corresponding to $105 \%$ of the maximal intensity reached in the GXT, which was used as verification testing to confirm $\dot{\mathrm{V}} \mathrm{O}_{2 \max }$ (Rossiter et al., 2006).
The $\dot{\mathrm{VO}}_{2}$ average of the final $30 \mathrm{~s}$ in each GXT stage and $15 \mathrm{~s}$ in the rectangular test was calculated. $\dot{\mathrm{V}} \mathrm{O}_{2 \max }$ was assumed when the $\dot{\mathrm{V}} \mathrm{O}_{2}$ plateau was observed (difference of $\leq 2.1 \mathrm{~mL} \cdot \mathrm{kg} \cdot \mathrm{min}^{-1}$ between the last two complete stages of GXT). When no plateau could be observed, the highest average of $\mathrm{VO}_{2}$ obtained during the GXT was compared with $\mathrm{VO}_{2}$ reached in the rectangular test; $\dot{\mathrm{V}} \mathrm{O}_{2 \max }$ being assumed as the highest average of $\dot{\mathrm{VO}}_{2}$ when not different from the $\dot{\mathrm{V}}_{2}$ reached in the rectangular test (Rossiter et al., 2006). The minimal exercise intensity at which the subject reached $\mathrm{V}_{2 \max }$ was considered as $\mathrm{i} \dot{\mathrm{V}} \mathrm{O}_{2 \max }$.

\section{Supplementation Protocol}

Twenty-four hours after the final familiarization test, the volunteers received $20 \mathrm{~g} \cdot$ day $^{-1}$ of placebo (dextrose) for 5 days for the "placebo" test session, followed by a 7 day "fake" washout period. Subsequently, creatine monohydrate (Creapure $^{\circledR}$, AlzChem AG, Germany, $20 \mathrm{~g} \cdot \mathrm{day}^{-1}$ ) was administered for another 5 days for the "creatine" test session. During supplementation and the washout period, the volunteers maintained their recreational exercise routine.

Supplements were given in 4 equal doses and the volunteers were instructed to ingest the supplements immediately after their main meal of the day. The placebo was given before creatine in a single blind design (i.e., only the participants were blinded) to avoid any carry over effect of creatine, considering its long-term washout period. The dose of creatine was chosen according to Harris et al. (1992) and Jacobs et al. (1997), who demonstrated that this dosage was sufficient to saturate the intramuscular creatine stores, and increase AC and time-to-exhaustion (tlim) in a supramaximal rectangular effort, respectively. Placebo and creatine supplements were identical in appearance, and were administered in flavored tablets containing $1 \mathrm{~g}$ of creatine and $2 \mathrm{~g}$ of dextrose each. At the end of the study, to test the efficacy of the blinding design, volunteers were asked about which arm of the study they had received the creatine supplement in. Only 5 out of the 14 volunteers correctly answered when they ingested creatine.

\section{Submaximal and Supramaximal Efforts}

Baseline $\dot{\mathrm{VO}}_{2}$ was measured with volunteers seated for $10 \mathrm{~min}$ before the tests. Subsequently, the volunteers performed five 10min submaximal efforts at $30,40,50,60$, and $70 \%$ of $\mathrm{iVO}_{2 \max }$, with the first three intensities performed as warm-up, $5 \mathrm{~min}$ before the supramaximal tests. The submaximal efforts at 60 and $70 \%$ of $\mathrm{iV} \mathrm{V}_{2 \max }$ were performed on different days, so as not to interfere with the performance of the supramaximal effort.

Supramaximal efforts at $115 \%$ were performed until voluntary exhaustion or the inability to continue the exercise, and the tlim was recorded. The choice of intensity of the supramaximal test (i.e., 115\%) was based on previous investigations which demonstrated that this intensity is the highest exercise intensity to determine the $\mathrm{AC}_{[\mathrm{La}-]+\mathrm{EPOCfast}}$ (Zagatto et al., 2016; Miyagi et al., 2017, 2018). Immediately after the end of the supramaximal tests, the participants remained seated quietly for $10 \mathrm{~min}$ for measurement of $\mathrm{EPOC}_{\text {fast }}$. The supramaximal efforts were performed 4 times, the first 2 efforts being used as familiarization and the next 2 efforts after the placebo and creatine ingestion periods. The final familiarization supramaximal test was 


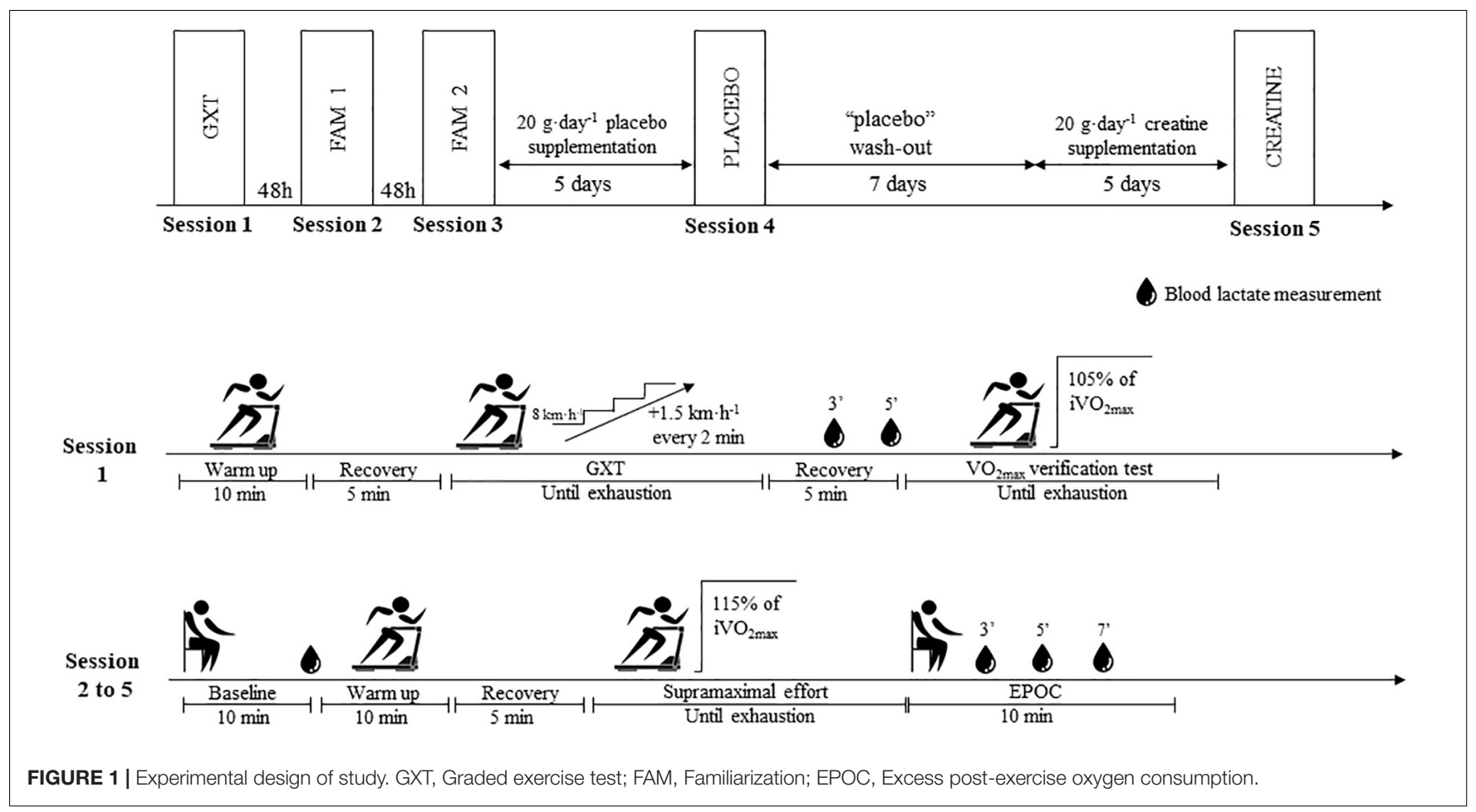

compared with the placebo condition to ensure that there was no longer any familiarization effect.

\section{Conventional MAOD Determination}

A linear regression was fitted using 5 submaximal intensities and respective $\dot{\mathrm{VO}_{2}}$, considering the $\dot{\mathrm{VO}}_{2}$ average 8-10 min, with the $y$-intercept fixed at the baseline $\dot{\mathrm{VO}}_{2}$ (Özyener et al., 2003). This linear regression was extrapolated to estimate the oxygen demand at $115 \%$ i $\dot{V} O_{2 \max }$ (Zagatto et al., 2016; Miyagi et al., 2017). In addition, the area of $\mathrm{VO}_{2}$ measured during the supramaximal intensity was determined using the trapezoidal method (Medbø et al., 1988; Zagatto et al., 2016; Miyagi et al., 2017). Therefore, the MAOD was considered as the difference between the estimated oxygen demand area (estimated oxygen demand at $115 \%$ of $\mathrm{iV} O_{2 \max }$ multiplied by tlim) and the area $\dot{\mathrm{VO}} 2$ of the supramaximal effort (Medbø et al., 1988).

TABLE 1 | GXT and rectangular exercise test parameters.

\section{Variables}

Exercise Duration (min)

Rectangular exercise duration (min)

$\dot{\mathrm{V}} \mathrm{O}_{2 \max }$ reached in GXT

$\left(\mathrm{mL} \cdot \mathrm{kg}^{-1} \cdot \mathrm{min}^{-1}\right)$

$\dot{\mathrm{V}}_{2 \text { max }}$ reached in rectangular test

$\left(\mathrm{mL} \cdot \mathrm{kg}^{-1} \cdot \mathrm{min}^{-1}\right)$

$\mathrm{iVO}_{2 \max }\left(\mathrm{km} \cdot \mathrm{h}^{-1}\right)$

Respiratory exchange ratio

$\left[\mathrm{La}^{-}\right]$PEAK $\left(\mathrm{mmol} \cdot \mathrm{L}^{-1}\right)$

Values are mean $\pm S D$ (C/95\%).

\section{$\mathrm{AC}_{[\mathrm{La}-]+\mathrm{EPOCfast}}$ and Non-mitochondrial Pathways Measurements}

The $\mathrm{AC}_{[\mathrm{La}-]+\text { EPOCfast }}$ was assumed as the sum of ePCr and $\mathrm{e}\left[\mathrm{La}^{-}\right]$estimated during the supramaximal effort (Bertuzzi et al., 2010; Zagatto et al., 2016, 2017a; Miyagi et al., 2017; Redkva et al., 2018). The ePCr was estimated by the product between $\mathrm{VO}_{2}$ amplitude (A1) and time constant ( $\left.\tau 1\right)$ from a first exponential decay fitted using a bi-exponential fit in $\mathrm{EPOC}_{\text {fast }}$, with OriginPro 8.0 software (Origin Lab Corporation, Microcal, MA, United States) (Bertuzzi et al., 2010; Zagatto et al., 2016, 2017a; Miyagi et al., 2017), while e[La $\left.{ }^{-}\right]$was estimated using the net lactate accumulation (i.e., difference between $[\mathrm{La}-]_{\text {peak }}$ and $\left.[\mathrm{La}-]_{B L}\right)$, considering for each $1 \mathrm{mmol} \cdot \mathrm{L}^{-1}$ of the net blood lactate an equivalent of $3 \mathrm{~mL} \cdot \mathrm{kg}^{-1}$ of oxygen (Di Prampero and Ferretti, 1999). The oxidative pathway (eOXID) was assumed as the $\dot{\mathrm{VO}}_{2}$ integral under the curve (i.e., $\dot{\mathrm{VO}}_{2}$ area) subtracting the baseline $\dot{\mathrm{V}} \mathrm{O}_{2}$ area.

\section{Statistical Analyses}

The data are presented as mean \pm standard deviation and 95\% confidence interval (CI95\%). All variables were examined using the Shapiro-Wilk test to check for normal distribution. To determine tlim reliability after familiarization, the intraclass correlation coefficients were applied (Koo and Li, 2016). The paired " $\mathrm{t}$ " test was used to compare the values of MAOD and $\mathrm{AC}_{[\mathrm{La}-]+\text { EPOCfast }}$ for each condition. Pearson's correlation test was also applied to determine the associations between values of MAOD and $\mathrm{AC}_{[\mathrm{La}-]+\text { EPOCfast }}$ for each condition. The Pearson's correlation test was applied and the coefficient of correlation was classified as negligible ( 0 to 0.2 ), weak ( $\geq 0.2$ to 0.4 ), moderate 

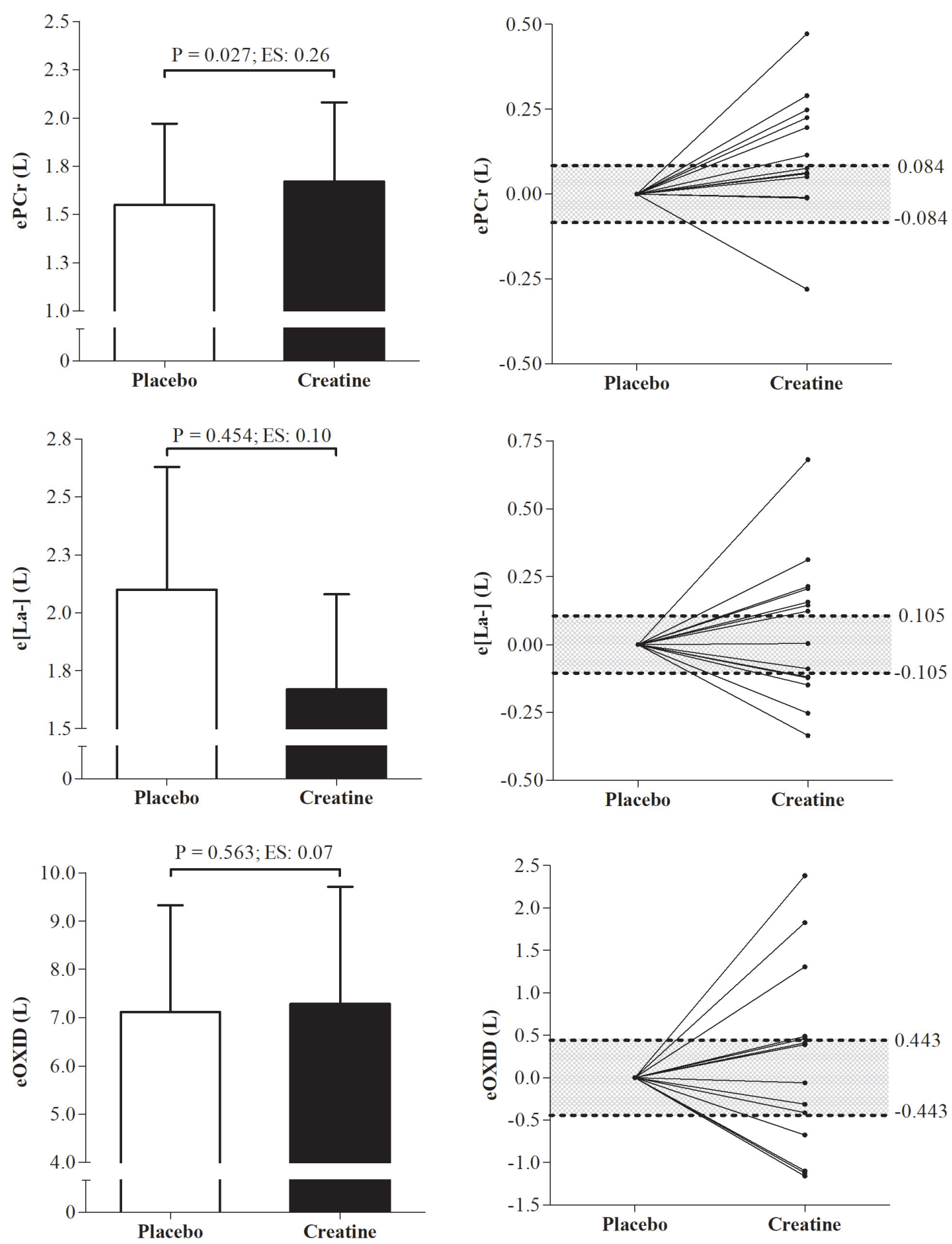

FIGURE 2 | Differences and individual smallest worthwhile change of energetics data from phosphagen, glycolytic, and oxidative pathways under placebo and creatine conditions. ePCr, energetics from the phosphagen systems; e[La'], energetics from glycolytic pathway; eOXID, oxidative phosphorylation pathway; ES, effect size.

( $\geq 0.4$ to 0.7 ), strong ( $\geq 0.7$ to 0.9 ), and very strong ( $\geq 0.9$ to 1.0) (Rowntree, 1991). In all cases, a significance level of $5 \%$ was assumed. As a qualitative analysis, the magnitude of differences between groups was calculated and expressed as standardized mean differences (Cohen's d), assuming threshold values for Cohen's d statistics of $\geq 0.2$ (small), $\geq 0.5$ (moderate), and $\geq 0.8$ 
(large) (Cohen, 1988). In all cases, a significance level of 5\% was assumed. The smallest worthwhile change was calculated as the product between the standard deviation between subjects in the placebo condition and 0.2 , to verify the change in substantial or harmful effect of creatine.

\section{RESULTS}

Peak and maximal variables measured during the GXT and verification testing are shown in Table 1 . The peak $\dot{\mathrm{VO}}_{2}$ measured during the GXT and during the verification testing at $105 \%$ to confirm the $\dot{\mathrm{V}} \mathrm{O}_{2 \max }$ was not different $(\mathrm{P}=0.19)$, confirming that $\dot{\mathrm{V}} \mathrm{O}_{2 \max }$ was measured.

Considering the values of $\mathrm{iV} \mathrm{O}_{2 \max }$ achieved in the GXT, the velocity corresponding to $115 \%$ applied during the supramaximal tests was $17.1 \pm 1.3(\mathrm{CI} 95 \%=16.3$ to 17.9$) \mathrm{km} \cdot \mathrm{h}^{-1}$. The tlim in the final familiarization supramaximal test was compared with the placebo condition, and demonstrated strong reliability ( ICC $=0.86 ; \mathrm{P}<0.001$ ).

The ePCr increased significantly in the creatine condition when expressed in absolute values $(P=0.027$; $\mathrm{ES}=0.26)$, besides which, 7 participants were responsive to creatine supplementation according to the smallest worthwhile change analysis. However, $\mathrm{e}\left[\mathrm{La}^{-}\right]$and eOXID were not altered $(\mathrm{P}=0.45$; $\mathrm{ES}=0.10$ and $\mathrm{P}=0.56 ; \mathrm{ES}=0.07$, respectively) even though 7 participants were responsive to creatine supplementation for these variables (Figure 2).

In addition, there were no differences between creatine and placebo conditions in tlim and in the ePCr, e[ $\left.\mathrm{La}^{-}\right]$, and eOXID when expressed in percentages of total energetics contribution (Table 2).

Figure 3 presents means $( \pm \mathrm{SD})$ and individual smallest worthwhile change values of $\mathrm{AC}$ measured using MAOD and $\mathrm{AC}_{[\mathrm{La}-]+E P O C f a s t}$ methods under creatine and placebo conditions. The AC measured by MAOD and $\mathrm{AC}_{[\mathrm{La}-]+\mathrm{EPOCfast}}$ did not present significant differences between placebo and creatine conditions $(\mathrm{P}=0.58$ and $\mathrm{P}=0.07)$. However, in the effect size (ES), the $\mathrm{AC}_{[\mathrm{La}-]+\text { EPOCfast }}$ showed a small positive effect of creatine supplementation on $\mathrm{AC}(\mathrm{ES}=0.20$;

TABLE 2 | Performance and percentage of metabolic energetics during the supramaximal effort in placebo and creatine conditions.

\begin{tabular}{lcccc}
\hline & Placebo & Creatine & $\boldsymbol{\Delta} \%$ & $\mathbf{P}$ \\
\hline $\operatorname{tlim}(\mathrm{s})$ & $160.79 \pm 37.76$ & $163.64 \pm 38.72$ & +2.27 & 0.49 \\
& $(138.98$ to 182.59$)$ & $(141.29$ to 186.00$)$ & & \\
\%e[La- $]$ & $19.89 \pm 3.17$ & $19.82 \pm 3.83$ & -0.06 & 0.91 \\
& $(18.06$ to 21.72$)$ & $(17.61$ to 22.03$)$ & & \\
\%ePCr & $15.09 \pm 4.69$ & $15.74 \pm 4.04$ & +0.65 & 0.25 \\
& $(12.38$ to 17.79$)$ & $(13.40$ to 18.07$)$ & & \\
\%eOXID & $65.02 \pm 6.20$ & $64.44 \pm 6.48$ & -0.59 & 0.49 \\
& $(61.44$ to 68.60$)$ & $(60.69$ to 68.18$)$ & &
\end{tabular}

Values of creatine and placebo condition are expressed as Mean $\pm S D$ (95\%/C). tlim, time to exhaustion; \%e[La-], percentage of energetics from glycolytic pathway; \%ePCr, percentage of energetics from the phosphagen systems; \%eOXID, percentage of energetics from oxidative phosphorylation pathway.
$\Delta \%=4.90 \%$ ), while the MAOD presented a negligible effect size $(\mathrm{ES}=0.08 ; \Delta \%=1.19 \%)$. Furthermore, 8 participants were positive responders to creatine supplementation for $\mathrm{AC}_{[\mathrm{La}-]+\text { EPOCfast }}$ and 5 for MAOD.

In addition, $\mathrm{MAOD}$ and $\mathrm{AC}_{[\mathrm{La}-]+E P O C f a s t}$ were not different $(\mathrm{P}=0.08)$ under the placebo condition and showed a moderate and significant correlation $(\mathrm{r}=0.68 ; \mathrm{P}=0.008)$. However, under the creatine condition, these variables also presented a significant and strong correlation $(\mathrm{r}=0.72 ; \mathrm{P}=0.003)$, although the $\mathrm{AC}$ measured using the $\mathrm{AC}_{[\mathrm{La}-]+E P O C f a s t}$ method was greater than the conventional MAOD procedure $(\mathrm{P}=0.02)$ (Figure 4).

When asked which supplement they took at each moment, only 5 out of the 14 volunteers correctly answered when they had ingested creatine, thus demonstrating that blinding of the study was effective for the majority of volunteers.

\section{DISCUSSION}

In the present study we investigated the effects of short-duration creatine monohydrate supplementation on AC measured using $\mathrm{AC}_{[\mathrm{La}-]+\text { EPOCfast }}$ and conventional MAOD methods. The main findings of the present study were the increase in ePCr and consequent small effect on AC measured by $\mathrm{AC}_{[\mathrm{La}-]+\mathrm{EPOCfast}}$, with 8 of 14 positive responders, despite the non-significant finding $(\mathrm{P}=0.07)$ Despite this, performance in the supramaximal effort at $115 \%$ of $\mathrm{iVO}_{2 \max }$ and $\mathrm{AC}$ measured by MAOD were not changed.

It is already known that monohydrated creatine shortduration supplementation may increase creatine intramuscular stores by $\sim 20 \%$, the phosphorylcreatine resynthesis rate, and ATP availability for the phosphagen pathway (Harris et al., 1992; Greenhaff et al., 1994; Terjung et al., 2000), thus being a possible mechanism to explain the increase in $\mathrm{PCr}$ verified in the present study. Although we did not measure creatine content in muscle and ATP availability, the rate of increase in ePCr corroborates previous findings, since even though the intramuscular content of creatine may increase at a higher rate (i.e., 10-20\%) after creatine administration, the energy supply increases at a lower rate (i.e., ranging from 2.5 to $10 \%$ ), being dependent on the intensity and duration of the effort (Terjung et al., 2000).

Despite the non-significant effect of creatine supplementation on $\mathrm{AC}$ measured by $\mathrm{AC}_{[\mathrm{La}-]+\text { EPOCfast }}$ and MAOD, a slight positive effect of creatine was verified only in $\mathrm{AC}_{[\mathrm{La}-]+\text { EPOCfast }}$ (ES $=0.20)$, indicating the responsiveness of this method, since the majority of participants were responsive to creatine administration (8 of 14 participants). Although non-significant, the slight increase could be directly attributed to the increase in ePCr, as the ePCr corresponds to approximately $40 \%$ of $\mathrm{AC}_{[\mathrm{La}-]+\mathrm{EPOCfast}}$ in moderately active individuals (Zagatto et al., 2017b). Jacobs et al. (1997) reported improvements in AC measured by the conventional MAOD method and in performance at $125 \%$ of $\mathrm{iVO}_{2 \max }$ intensity, after 5 days of creatine supplementation $\left(20 \mathrm{~g} \cdot \mathrm{day}^{-1}\right)$, not supporting our results (i.e., no differences in MAOD or tlim). However, Doherty et al. (2002), using the same intensity, did not find differences for the MAOD or tlim, corroborating with the results of the present study. 

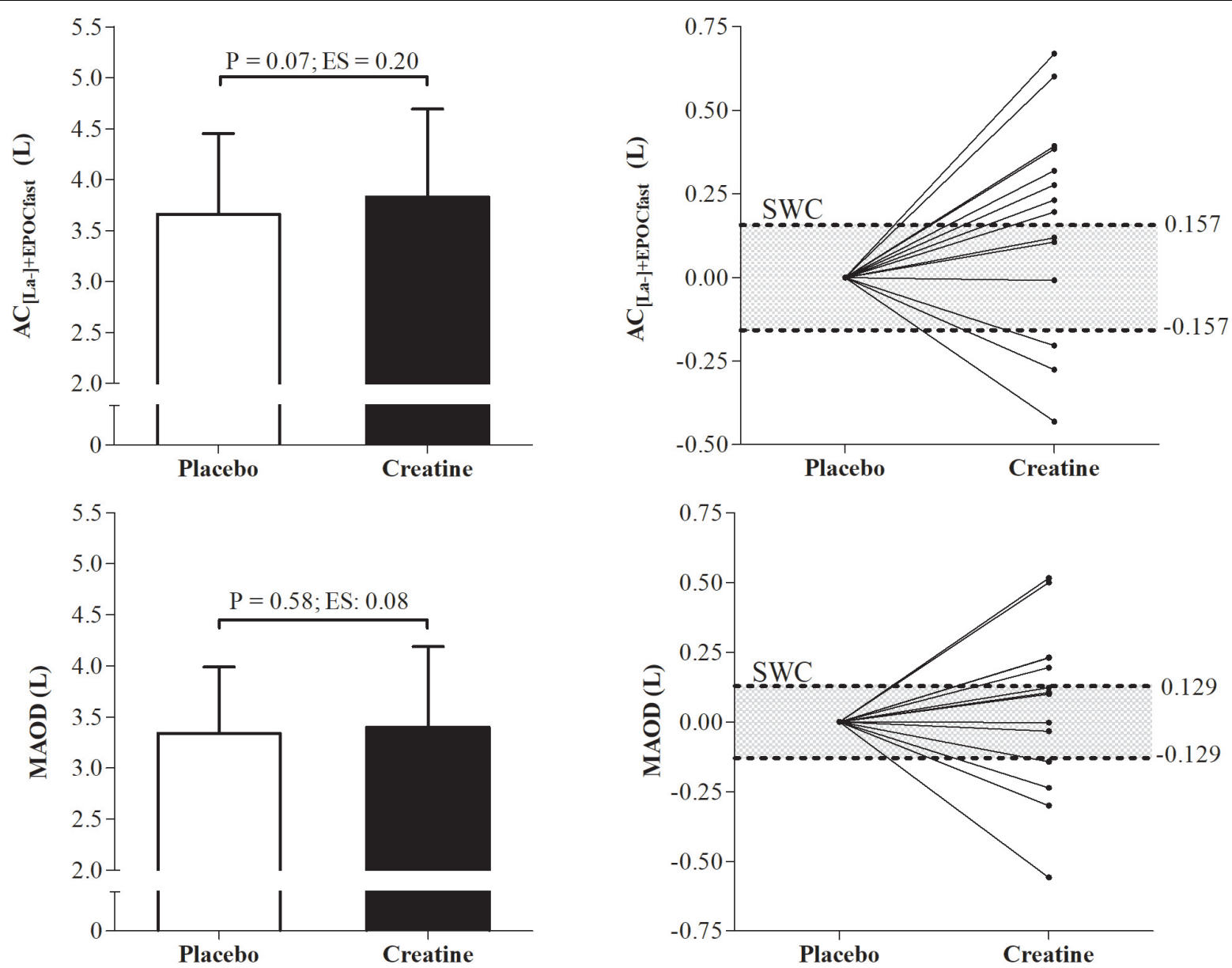

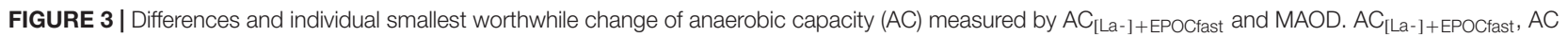
estimated by non-oxidative energetics sum measured during a single supramaximal effort; MAOD, maximal accumulated oxygen deficit; ES, Effect size; SWC, Smallest worthwhile change.

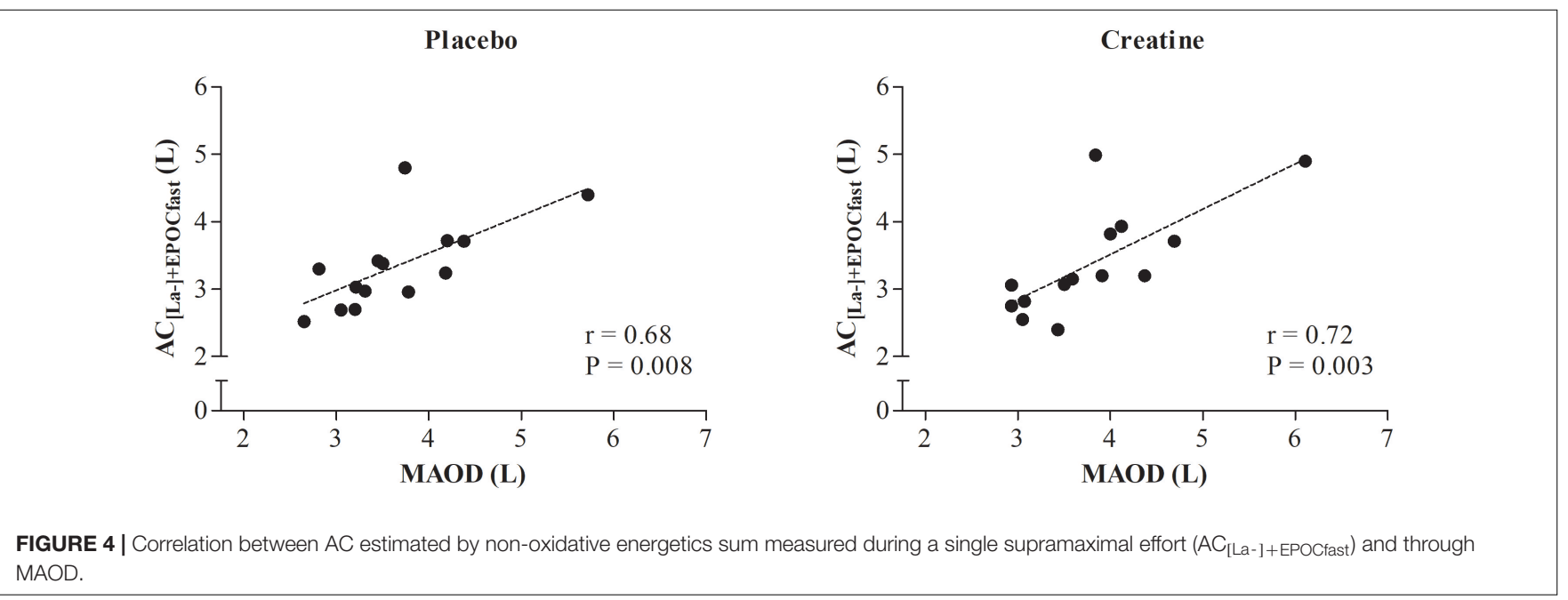

Although MAOD is considered the most accepted method for AC measurement (Medbø and Tabata, 1993; Noordhof et al., 2010), a possible explanation for the discordant results could be supported by the high values of limits of agreement $\left(15.1 \mathrm{~mL} \cdot \mathrm{kg}^{-1}\right)$ and poor reliability of the conventional MAOD method, making a larger sample size necessary to 
detect small differences (Doherty et al., 2000). On the other

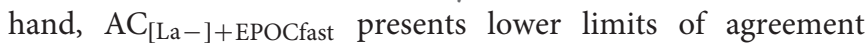
$\left(2.9 \mathrm{~mL} \cdot \mathrm{kg}^{-1}\right)$ and coefficient of variation $(4.1 \%)$ values (Zagatto et al., 2016; Miyagi et al., 2017), thus possibly explaining why $\mathrm{AC}_{[\mathrm{La}-]+\mathrm{EPOCfast}}$, although with no significant change $(\mathrm{P}=0.07)$, was able to detect a small positive impact of creatine supplementation compared with the placebo condition $(\mathrm{ES}=0.20)$, differing from the MAOD result $(\mathrm{P}=0.58 ; \mathrm{ES}=0.08)$. In addition, recently Brisola et al. (2015) and de Poli et al. (2016) verified improvement in $\mathrm{AC}_{[\mathrm{La}-]+\text { EPOCfast }}$ caused by acute sodium bicarbonate and caffeine supplementation, suggesting the "efficiency" of the method to detect slight changes caused by ergogenic sources. However, it should be mentioned that the possibly higher sensitivity of $\mathrm{AC}_{[\mathrm{La}-]+\text { EPOCfast }}$ when compared with MAOD has to be assumed carefully, since our results present a small effect on this variable. Thus, further studies are necessary to elucidate the sensitivity of $\mathrm{AC}_{[\mathrm{La}-]+E P O C f a s t}$ and MAOD to detect changes caused by creatine intake compared with a direct method (i.e., muscle biopsy). In addition, both the placebo and creatine conditions showed a significant and moderate correlation between $\mathrm{AC}_{[\mathrm{La}-]+\text { EPOCfast }}$ and MAOD ( $r=0.68$ and 0.72 , respectively), corroborating with the results of Zagatto et al. (2016) $(r=0.73)$ and Miyagi et al. (2017) $(r=0.68)$. It should also be mentioned that the significant difference between $\mathrm{AC}_{[\mathrm{La}-]+\text { EPOCfast }}$ and MAOD under the creatine condition $(P=0.02)$ added to the maintenance of a moderate correlation between the methods.

Despite the increase in ePCr and the small effect on $\mathrm{AC}$ measured by $\mathrm{AC}_{[\mathrm{La}-]+E P O C f a s t}$ after monohydrated creatine supplementation, performance in the supramaximal effort was not changed. This result is also divergent from those reported by Jacobs et al. (1997), which could be largely attributed to the supramaximal intensity, since we used $115 \%$ of $\mathrm{iV} \mathrm{O}_{2 \max }$ (i.e., scientifically backed assessment for $\mathrm{AC}_{[\mathrm{La}-]+\mathrm{EPOCfast}}$ determination) (Zagatto et al., 2016; Miyagi et al., 2017), and Jacobs et al. (1997) used $125 \%$ of $\mathrm{iVO}_{2 \max }$, which could increase the portion of the energy supply by ePCr during the effort and its importance for the performance (Terjung et al., 2000; Mesa et al., 2002), as the present study showed that ePCr supplies a small portion of the total energy during the $115 \%$ of $\mathrm{iVO}_{2 \max }$ effort (Table 2).

In addition, the values of $\mathrm{e}\left[\mathrm{La}^{-}\right]$were not altered after creatine supplementation. This result was expected as there is no evidence that creatine supplementation increases the glycogen content or glycolysis activity, corroborating the results

\section{REFERENCES}

Bemben, M. G., and Lamont, H. S. (2005). Creatine supplementation and exercise performance recent findings. Sports Med. 35, 107-125. doi: 10.2165/00007256200535020-00002

Bertuzzi, R. C. M., Franchini, E., Ugrinowitsch, C., Kokubun, E., Lima-Silva, A. E., Pires, F. O., et al. (2010). Predicting MAOD using only a supramaximal exhaustive test. Int. J. Sports Med. 31, 477-481. doi: 10.1055/s-0030- 1253375

Brisola, G. M. P., Miyagi, W. E., da Silva, H. S., and Zagatto, A. M. (2015). Sodium bicarbonate supplementation improved MAOD but is not correlated with 200- of Doherty et al. (2002), although Zuniga et al. (2012) reported an increase in ATP production through the non-oxidative glycolytic pathway in the Wingate anaerobic test after creatine supplementation, attributing this result to the creatine buffering effect. The eOXID also remained unchanged, probably due to the lack of improvement in performance.

The main limitation of the present study was the lack of randomization of the tests. However, it is noteworthy that although no randomization was performed, two familiarizations were carried out before starting all procedures to minimize a possible "learning effect," and during the test, no difference was verified between the last familiarization and the placebo condition. Therefore, despite this being a study limitation, the lack of randomization seems not to have affected our findings.

\section{CONCLUSION}

In summary, short-term monohydrated creatine supplementation (20 $\mathrm{g} \cdot \mathrm{day}^{-1}$ for 5 days) improves the ePCr, although it is not enough to significantly improve the $\mathrm{AC}$ measured by $\mathrm{AC}_{[\mathrm{La}-]+\text { EPOCfast }}$ and $\mathrm{MAOD}$, or performance during a supramaximal effort.

\section{AUTHOR CONTRIBUTIONS}

RdP collected and analyzed the data, and wrote the manuscript. LR collected the data. EM wrote the manuscript. GA, RB, and $\mathrm{AZ}$ conceived the idea, built the experimental design, and wrote the manuscript.

\section{FUNDING}

RdP and EM were supported by São Paulo Research Foundation (FAPESP) fellowship (\#2016/17836-2 and \#2017/21724-8, respectively). This study was financed in part by the Coordenação de Aperfeiçoamento de Pessoal de Nível Superior - Brasil (CAPES) - Finance Code 001.

\section{ACKNOWLEDGMENTS}

The authors would like to thank the Prof. Bruno Gualano, Ph.D., for reviewing this study.

and 400-m running performances: a double-blind, crossover, and placebocontrolled study. Appl. Physiol. Nutr. Metab. 40, 931-937. doi: 10.1139/apnm2015-0036

Cohen, J. (1988). Statistical Power Analysis for the Behavioral Sciences, 2nd Edn. Hillsdale, NJ: Lawrence Erlbaum Associates.

de Poli, R. D. A. B., Miyagi, W. E., Nakamura, F. Y., and Zagatto, A. M. (2016). Caffeine improved time to exhaustion but did not change alternative maximal accumulated oxygen deficit estimated during a single supramaximal running bout. Int. J. Sport Nutr. Exerc. Metab. 26, 549-557. doi: 10.1123/ijsnem.20160038 
Di Prampero, P. E., and Ferretti, G. (1999). The energetics of anaerobic muscle metabolism: a reappraisal of older and recent concepts. Respir. Physiol. 118, 103-115. doi: 10.1016/S0034-5687(99)00083-3

Doherty, M., Smith, P. M., and Schroder, K. (2000). Reproducibility of the maximum accumulated oxygen deficit and run time to exhaustion during shortdistance running. J. Sports Sci. 18, 331-338. doi: 10.1080/026404100402395

Doherty, M., Smith, P. M., Davison, R. C. R., and Hughes, M. G. (2002). Caffeine is ergogenic after supplementation of oral creatine monohydrate. Med. Sci. Sports Exerc. 34, 1785-1792. doi: 10.1097/00005768-200211000-00015

Greenhaff, P., Bodin, K., Soderlund, K., and Hultman, E. (1994). Effect of oral creatine supplementation on skeletal muscle phosphocreatine resynthesis. Am. J. Physiol. 266(5 Pt 1), E725-E7230. doi: 10.1152/ajpendo.1994.266.5.E725

Gualano, B., Artioli, G. G., Poortmans, J. R., and Lancha Junior, A. H. (2010). Exploring the therapeutic role of creatine supplementation. Amino Acids 38, 31-44. doi: 10.1007/s00726-009-0263-6

Hall, M., and Trojian, T. H. (2013). Creatine supplementation. Curr. Sports Med. Rep. 12, 240-244. doi: 10.1249/JSR.0b013e31829cdff2

Harris, R. C., Söderlund, K., and Hultman, E. (1992). Elevation of creatine in resting and exercised muscle of normal subjects by creatine supplementation. Clin. Sci. 83, 367-374. doi: 10.1042/cs0830367

Hill, D. W. (2014). Morning-evening differences in response to exhaustive severeintensity exercise. Appl. Physiol. Nutr. Metab. 39, 248-254. doi: 10.1139/apnm2013-0140

Howley, E. T., Bassett, D. R., and Welch, H. G. (1995). Criteria for maximal oxygen uptake: review and commentary. Med. Sci. Sports Exerc. 27, 1292-1301. doi: 10.1249/00005768-199509000-00009

Hultman, E., Söderlund, K., Timmons, J. A., Cederblad, G., and Greenhaff, P. L. (1996). Muscle creatine loading in men. J. Appl. Physiol. 81, 232-237. doi: 10.1152/jappl.1996.81.1.232

Jacobs, I., Bleue, S., and Goodman, J. (1997). Creatine ingestion increases anaerobic capacity and maximum accumulated oxygen deficit. Can. J. Appl. Physiol. 22, 231-243. doi: 10.1139/h97-015

Jones, A. M., and Doust, J. H. (1996). A 1\% treadmill grade most accurately reflects the energetic cost of outdoor running. J. Sports Sci. 14, 321-327. doi: 10.1080/02640419608727717

Koo, T. K., and Li, M. Y. (2016). A guideline of selecting and reporting intraclass correlation coefficients for reliability research. J. Chiropr. Med. 15, 155-163. doi: $10.1016 /$ j.jcm.2016.02.012

Medbø, J. I., and Tabata, I. (1993). Anaerobic energy release in working muscle during $30 \mathrm{~s}$ to $3 \mathrm{~min}$ of exhausting bicycling. J. Appl. Physiol. 75, 1654-1660. doi: 10.1152/jappl.1993.75.4.1654

Medbø, J. I., Mohn, A. C., Tabata, I., Bahr, R., Vaage, O., and Sejersted, O. M. (1988). Anaerobic capacity determined by maximal accumulated $\mathrm{O} 2$ deficit. J. Appl. Physiol. 64, 50-60. doi: 10.1152/jappl.1988.64.1.50

Mesa, J. L. M., Ruiz, J. R., González-Gross, M. M., Gutiérrez Sáinz, A., and Castillo Garzón, M. J. (2002). Oral creatine supplementation and skeletal muscle metabolism in physical exercise. Sports Med. 32, 903-944. doi: 10.2165/ 00007256-200232140-00003

Miyagi, W. E., Bertuzzi, R., Nakamura, F. Y., de Poli, R. A. B., and Zagatto, A. M. (2018). Effects of caffeine ingestion on anaerobic capacity in a single supramaximal cycling test. Front. Nutr. 5:86. doi: 10.3389/fnut.2018.00086

Miyagi, W. E., de Poli, R. A. B., Papoti, M., Bertuzzi, R., and Zagatto, A. M. (2017). Anaerobic capacityestimated in a single supramaximal test in cycling: validity and reliability analysis. Sci. Rep. 7:42485. doi: 10.1038/srep42485

Noordhof, D. A., De Koning, J. J., and Foster, C. (2010). The maximal accumulated oxygen deficit method: a valid and reliable measure of anaerobic capacity? Sports Med. 40, 285-302. doi: 10.2165/11530390-000000000-00000
Özyener, F., Rossiter, H. B., Ward, S. A., and Whipp, B. J. (2003). Negative accumulated oxygen deficit during heavy and very heavy intensity cycle ergometry in humans. Eur. J. Appl. Physiol. 90, 185-190. doi: 10.1007/s00421003-0870-y

Padulo, J., Chamari, K., and Ardigò, L. P. (2014). Walking and running on treadmill: the standard criteria for kinematics studies. Muscles Ligaments Tendons J. 4, 159-162. doi: 10.32098/mltj.02.2014.13

Persky, A., and Brazeau, G. (2001). Clinical pharmacology of the dietary supplement creatine monohydrate. Pharmacol. Rev. 53, 161-176.

Redkva, P. E., Miyagi, W. E., Milioni, F., and Zagatto, A. M. (2018). Anaerobic capacity estimated by the sum of both oxygen equivalents from the glycolytic and phosphagen pathways is dependent on exercise mode: running versus cycling. PLoS One 13:e0203796. doi: 10.1371/journal.pone.0203796

Rossiter, H., Kowalchuk, J., and Whipp, B. (2006). A test to establish maximum $\mathrm{O} 2$ uptake despite no plateau in the $\mathrm{O} 2$ uptake response to ramp incremental exercise. J. Appl. Physiol. 100, 764-770. doi: 10.1152/japplphysiol.00932.2005

Rowntree, D. (1991). Statistics Without Tears?: A Primer for Non-Mathematicians. London: Penguin Books.

Terjung, R. L., Clarkson, P., Eichner, E. R., Greenhaff, P. L., Hespel, P. J., Israel, R. G., et al. (2000). American college of sports medicine roundtable. The physiological and health effects of oral creatine supplementation. Med. Sci. Sports Exerc. 32, 706-717. doi: 10.1097/00005768-200003000-00024

Volek, J. S., and Rawson, E. S. (2004). Scientific basis and practical aspects of creatine supplementation for athletes. Nutrition 20, 609-614. doi: 10.1016/j.nut. 2004.04.014

Yquel, R. J., Arsac, L. M., Thiaudière, E., Canioni, P., and Manier, G. (2002). Effect of creatine supplementation on phosphocreatine resynthesis, inorganic phosphate accumulation and $\mathrm{pH}$ during intermittent maximal exercise. J. Sports Sci. 20, 427-437. doi: 10.1080/026404102317366681

Zagatto, A. M., Bertuzzi, R., Miyagi, W. E., Padulo, J., and Papoti, M. (2016). MAOD determined in a single supramaximal test: a study on the reliability and effects of supramaximal intensities. Int. J. Sports Med. 37, 700-707. doi: 10.1055/s-0042-104413

Zagatto, A. M., Miyagi, W. E., De Barros Sousa, F. A., and Gobatto, C. A. (2017a). Relationship between anaerobic capacity estimated using a single effort and 30-s tethered running outcomes. PLoS One 12:e0172032. doi: 10.1371/journal.pone. 0172032

Zagatto, A. M., Nakamura, F. Y., Milioni, F., Miyagi, W. E., de Poli, R. A. B., Padulo, J., et al. (2017b). The sensitivity of the alternative maximal accumulated oxygen deficit method to discriminate training status. J. Sports Sci. 35, 24532460. doi: 10.1080/02640414.2016.1273539

Zuniga, J. M., Housh, T. J., Camic, C. L., Hendrix, C. R., Mielke, M., Johnson, G. O., et al. (2012). The effects of creatine monohydrate loading on anaerobic performance and one-repetition maximum strength. J. Strength Cond. Res. 26, 1651-1656. doi: 10.1519/JSC.0b013e318234eba1

Conflict of Interest Statement: The authors declare that the research was conducted in the absence of any commercial or financial relationships that could be construed as a potential conflict of interest.

Copyright (c) 2019 de Poli, Roncada, Malta, Artioli, Bertuzzi and Zagatto. This is an open-access article distributed under the terms of the Creative Commons Attribution License (CC BY). The use, distribution or reproduction in other forums is permitted, provided the original author(s) and the copyright owner(s) are credited and that the original publication in this journal is cited, in accordance with accepted academic practice. No use, distribution or reproduction is permitted which does not comply with these terms. 